\title{
¿QUIÉN ES LA VÍCTIMA Y QUIÉN EL AGRESOR EN LA VIOLENCIA FÍSICA ENTRE PAREJAS? ESTUDIO EPIDEMIOLÓGICO EN SIETE CIUDADES DEL PERÚ
}

\author{
Fabián Fiestas ${ }^{1,2, a}$, Ruth Rojas ${ }^{3, b}$, Alfonso Gushiken ${ }^{4, c}$, Ernesto Gozzer ${ }^{5, d}$
}

\begin{abstract}
RESUMEN
Objetivos. Identificar factores asociados con la probabilidad de ser víctima o agresor en violencia física entre parejas. Materiales y métodos. Se analizó la base de datos de una encuesta poblacional aplicada en siete ciudades de Perú (Lima, Arequipa, Huamanga, Trujillo, Cusco, Callao y Maynas). Participaron 6399 hombres y mujeres, de los cuales 3909 refirieron vivir en pareja al momento de la entrevista. Se empleó regresión logística univariada y multivariada para identificar factores asociados con cualquiera de los roles, víctima o agresor. El alfa establecido fue 0,01. Resultados. La probabilidad de ser víctima o agresor en la violencia física entre parejas fue similar para ambos sexos $(p>0,01)$, e independiente del tiempo de convivencia. La exposición a la violencia en la niñez, tener una actitud favorable a la violencia, ser menor de 45 años, el consumo excesivo de alcohol, tener educación superior incompleta y tener un ingreso familiar de menos de 750 USD son factores asociados tanto a ser víctima como a ser agresor. Geográficamente, Cusco es la ciudad en que se puede encontrar con mayor probabilidad personas que ejercen violencia física contra la pareja o son víctimas de ella. El valor fue $p<0,001$ para la mayoría de estas asociaciones. Conclusiones. El patrón de características que predice quién es la víctima, es muy similar al que predice quién es el agresor en los casos de violencia física contra la pareja. El sexo y los años de convivencia no son predictivos para ninguno de los roles violencia física contra la pareja.
\end{abstract}

Palabras clave: Violencia basada en género; Maltrato conyugal; Epidemiología; Perú (fuente: DeCS BIREME).

\section{WHO IS THE VICTIM AND WHO THE OFFENDER IN INTIMATE PARTNER PHYSICAL VIOLENCE? AN EPIDEMIOLOGICAL STUDY IN SEVEN CITIES OF PERU}

\begin{abstract}
Objectives. To identify factors associated to the probability of being the aggressor or the victim in cases of intimate partner physical violence. Materials and methods. A secondary data analysis was performed to an epidemiological survey done in seven cities in Peru (Lima, Arequipa, Huamanga, Trujillo, Cuzco, Callao and Maynas). 6399 men and women participated, of whom 3909 participants declared living together with an intimate partner at the time of the interview. Univariate and multivariate logistic regression models were used to identify associated factors to the roles of victim or aggressor. Results. The likelihood of being aggressor or victim in cases of intimate partner physical violence was independent of $\operatorname{sex}(p<0,01)$ and the couple's time living together. Factors associated to a higher probability of both roles included a history of exposure to physical violence during childhood, having a favorable attitude towards violence, heavy alcohol drinking, and being younger than 45 years, as well as having a monthly family income of less than 750 USD or an educational attainment of less than complete tertiary school. Geographically, Cusco was the city where it was most likely to find an aggressor or a victim of intimate partner physical violence. Most of these associations obtained p-values of less than 0.001 . Conclusions. In cases of intimate partner physical violence, the pattern of predictors is similar in both roles, i.e., aggressor and victim. Sex and the couple's span of time living together did not predict any of both roles.
\end{abstract}

Key words: Violence based on gender; Spouse abuse; Epidemiology; Peru (source: MeSH NLM).

\footnotetext{
Unidad de Análisis y Generación de Evidencias en Salud Pública, Instituto Nacional de Salud. Lima, Perú.

Red para la Acción y Avance de la Salud Mental y Psiquiatría (Red AVANSE-PSI). Lima, Perú.

3 Laboratorio de Neurobiología Molecular, Laboratorios de Investigación y Desarrollo, Universidad Peruana Cayetano Heredia. Lima, Perú.

4 Facultad de Salud Pública y Administración, Universidad Peruana Cayetano Heredia. Lima, Perú.

Universidad Peruana Cayetano Heredia. Lima, Perú.

a Médico epidemiólogo; ${ }^{\mathrm{b}}$ bióloga; ${ }^{\mathrm{c}}$ médico, magíster en salud pública, magíster en ciencias sociales; ${ }^{\mathrm{d}}$ médico salubrista, especialista en salud internacional
} 


\section{INTRODUCCIÓN}

La violencia doméstica es un problema muy frecuente que ha llevado a una serie de organismos de la sociedad civil y gobiernos a promover políticas públicas para reducir su frecuencia e impacto sobre la sociedad ${ }^{(1,2)}$. En un estudio realizado por la Organización Mundial de la Salud en diez países, se encontró que la prevalencia de vida de violencia física, sexual o ambas, variaba de 15 a $71 \%$ y que en Perú, el $42 \%$ de la mujeres de $15-49$ años sufrieron violencia física al menos una vez en su vida de pareja ${ }^{(3)}$. Según la Encuesta Nacional de Demografía y Salud Familiar de 2010, 38,4\% de las mujeres de 15 a 49 años que había estado alguna vez unida había sido empujada, golpeada o agredida físicamente por su esposo o compañero ${ }^{(4)}$. En Lima, según un estudio realizado por Güezmes et al., esta cifra llegó a $48 \%$, mientras que en Cusco se elevó a 60,9\% ${ }^{(5)}$. La violencia de pareja puede terminar en asesinato de la mujer ${ }^{(6)}$; así, Gushiken et al., con datos de la División de Homicidios de la Policía Nacional del Perú, informaron que el $12 \%$ de los homicidios ocurridos en Lima entre 2000-2008 fueron cometidos por la pareja, cifra que alcanzó casi el $40 \%$ en los casos de homicidios de mujeres ${ }^{(7)}$.

Pese a su magnitud e importancia, existen pocos estudios poblacionales peruanos que exploren en profundidad este tema y que generen información concreta para localizar a grupos de mayor riesgo y para identificar sus mecanismos y causas. A ello se suma la gran dispersión de informes y subregistro en los sistemas de información oficiales, todo lo cual ocasiona que los tomadores de decisiones finalmente tengan poca evidencia contextualizada para orientar las políticas, programas o intervenciones.

En este contexto, y con el objeto de identificar las creencias, actitudes y prácticas sobre violencia, que proporcionen insumos para el diseño de políticas y programas preventivos, de educación y comunicación social, la Secretaría Técnica del Consejo Nacional de Seguridad Ciudadana del Perú (CONASEC), en el marco de la preparación del Programa Consolidación Democrática de la Seguridad Ciudadana, encargó al Instituto Cuánto la recolección de datos de la encuesta "Creencias, Actitudes y Comportamientos sobre Violencia" en nueve distritos de Lima y seis ciudades del interior del país. Esta encuesta tuvo como base el protocolo e instrumentos elaborados por el proyecto ACTIVA, ejecutado por la OPS/OMS en diez ciudades de América Latina y España ${ }^{(8)}$ y sirvió como fuente para identificar factores asociados a la violencia.

Sin dejar de reconocer la naturaleza multicausal y compleja del fenómeno y la diversidad de actitudes que orientan la conducta humana, esta investigación intenta seleccionar información que los tomadores de decisiones puedan usar para pasar a la acción. Así, para este estudio se escogieron, basados en el marco conceptual de asociación, solo tres tipos de variables entre las contenidas en la base de datos: a) variables con capacidad de localizar a quienes están en mayor riesgo de ser víctimas o agresores (sexo, edad, tiempo de convivencia, nivel educativo e ingreso familiar); b) variables susceptibles de ser modificadas con intervenciones sociales o políticas públicas (el haber sido víctima de violencia física durante la niñez, el consumo excesivo y frecuente de alcohol, la actitud positiva hacia la violencia); y c) el lugar de residencia del participante, debido a que hay informes en los que se encontró que la frecuencia y el nivel de violencia son mayores en algunos lugares que en otros, especialmente en lugares ubicados en la sierra peruana.

En resumen, el presente estudio tiene como objetivo identificar factores asociados, tanto con la probabilidad de ser víctimas como de ser agresor de violencia física entre parejas.

\section{MATERIALES Y MÉTODOS}

Se realizó el análisis secundario de los datos de la encuesta del CONASEC, el cual fue un estudio transversal cuya población la constituyeron los residentes de los hogares de Lima Metropolitana (incluyó nueve distritos: Cercado de Lima, Comas, Rímac, San Juan de Lurigancho, San Martín de Porres, Surco, Villa María del Triunfo, Surquillo y Villa el Salvador) y seis ciudades del interior del país (Tabla 1). La encuesta fue aplicada entre mayo y junio de 2006 a una muestra de personas de ambos sexos entre 15 a 70 años.

El cálculo del tamaño de muestra total siguió los parámetros establecidos por el Proyecto ACTIVA (8). La muestra se estableció considerando un nivel de confianza de $95 \%$ en la estimación de proporciones poblacionales, con un error máximo permitido entre 4,5 y $7,1 \%$. Con ello, resultó en un tamaño de muestra de aproximadamente 400 personas por cada distrito de Lima Metropolitana y cada ciudad incluida. Al proponerse un diseño sin sustitución, se incrementó la muestra en $10 \%$, considerando la tasa de rechazos. La muestra total fue de 6399 personas (2592 varones y 3807 mujeres).

\section{EVALUACIONES E INSTRUMENTOS}

Se utilizó el cuestionario ACTIVA ${ }^{(8)}$, que incluye preguntas que abordan las características sociodemográficas de 
los entrevistados y sus familias, las actitudes frente a comportamientos violentos y frente a las normas, las habilidades para emitir respuestas alternativas a la violencia, la percepción de las instituciones sociales y de gobierno, el consumo excesivo de alcohol, el uso de medios de comunicación y la prevalencia de comportamientos violentos, tanto en la familia como en la comunidad.

Las variables dependientes del estudio fueron: i) agresor, que denota que el participante ejerció violencia física sobre la pareja (golpes con cachetadas, puño, patadas o con objetos) durante los últimos doce meses; y ii) víctima de violencia física, si el participante había recibido golpes con cachetadas, puño, patadas o con objetos, por parte de su pareja durante los últimos doce meses.

Como variables independientes se tomaron: el lugar de residencia, sexo, edad, tiempo de convivencia, grado de instrucción, el haber sido víctima de violencia física durante la niñez (es decir, si recibió castigos físicos, incluyendo jalones de pelo o de orejas, palmadas en las manos o nalgas, golpes con el puño, pie o con algún objeto), el consumo excesivo de alcohol (definido como haber ingerido ocho vasos de cerveza o cinco de licor en una sola ocasión) durante el mes previo y tener una actitud favorable hacia la violencia (variable continua dividida en cuartiles, donde el cuartil superior denota una actitud más positiva hacia la violencia).

\section{PROCEDIMIENTOS}

Para la selección de la muestra se empleó el muestreo probabilístico, estratificado, polietápico, en el que las unidades primarias de selección fueron las manzanas (primera etapa), luego las viviendas (segunda etapa) y finalmente las personas (tercera etapa).

Aunque este fue un estudio de diseño poblacional con muestreo multietápico, probabilístico, las variables que dan cuenta de este diseño no pudieron ser obtenidas por los autores de este estudio y, por ello, todas las estimaciones estadísticas aquí presentadas son datos no ponderados, lo que impone cierta limitación a la interpretación de algunos de los estimados estadísticos calculados, como se explica en el análisis de datos.

El proyecto contó con la aprobación del Ministerio del Interior y su ejecución fue supervisada por el equipo técnico a cargo del Proyecto y la Secretaría Técnica del CONASEC. Se solicitó el consentimiento verbal de cada entrevistado.

\section{ANÁLISIS DE DATOS}

Para efectos del presente estudio, solo se trabajó con los 3909 hombres y mujeres participantes que refirieron vivir actualmente en pareja (últimos 12 meses). El análisis inicial incluyó una descripción de frecuencias y porcentajes no ponderados de las variables categóricas, y las distribuciones y medidas de tendencia central de las variables continuas. Luego, se realizó la estimación de las medidas de asociación entre las variables independientes con el hecho de ser agresor o víctima de violencia física contra la pareja, fue realizado usando regresión logística univariada y multivariada. La regresión logística univariada estimó los Odds Ratio (OR) crudos de asociación entre ser víctima y, luego, entre ser agresor y con cada una de las nueve variables independientes consideradas para este estudio.

El análisis multivariado implicó la estimación de OR de un modelo donde todas las variables independientes son consideradas simultáneamente, excepto en el modelo que valora el efecto de ingreso mensual familiar, en el cual no incluía nivel de educación y viceversa, para evitar la posibilidad de colinearidad estadística entre ambas variables, dado que una está estrechamente correlacionada con la otra, tanto teóricamente como empíricamente en nuestra muestra $(p<0,001)$.

Debido a que no se contó con las variables que dan cuenta de la probabilidad de selección de los sujetos y del muestreo multietápico, no se pudo utilizar la regresión logística para muestras complejas, sino que se usó una regresión logística para muestras aleatorias simples. Para evitar con la mayor probabilidad de cometer error tipo I, que se da cuando no se toma en cuenta la complejidad del muestreo, se decidió establecer un valor $p<0,01$ para señalar significancia estadística. Los análisis fueron realizados en el programa STATA 11.0.

\section{RESULTADOS}

De las 6399 personas que participaron en el estudio, 3909 informaron estar viviendo actualmente con su esposo o compañero. La distribución no ponderada de las características de las personas que conviven con pareja es presentada por rol (agresor o víctima) en la Tabla 1.

Lo más importante de notar en la Tabla 1, es que para las categorías de las características estudiadas existe un número razonable de individuos como para permitir el modelamiento estadístico multivariado $(n>100)$, excepto para la categoría de personas menores de 18 años $(n=11)$ y personas con consumo excesivo de alcohol 
Tabla 1. Distribución de características por rol (agresor o víctima) en casos de violencia contra la pareja.

\begin{tabular}{|c|c|c|c|c|c|}
\hline \multirow[b]{2}{*}{ Variable } & \multirow{2}{*}{$\begin{array}{l}\text { Total } \\
(\mathbf{1 0 0 \% )}\end{array}$} & \multicolumn{2}{|c|}{ Agresor } & \multicolumn{2}{|c|}{ Víctima } \\
\hline & & $\begin{array}{c}\mathrm{Si} \\
\mathrm{n}(\%)\end{array}$ & $\mathbf{p}^{*}$ & $\begin{array}{c}\mathrm{Si} \\
\mathrm{n}(\%)\end{array}$ & $\mathbf{p}^{*}$ \\
\hline \multicolumn{6}{|l|}{ Sexo } \\
\hline Femenino & 2325 & $329(14,2)$ & 0,039 & $286(12,3)$ & 0,558 \\
\hline Masculino & 1585 & $188(11,9)$ & & $185(11,7)$ & \\
\hline \multicolumn{6}{|l|}{ Edad (años) } \\
\hline De 60 a más & 455 & $20(4,4)$ & $<0,001$ & $24(5,3)$ & $<0,001$ \\
\hline De 59-46 & 1006 & $81(8,1)$ & & $83(8,2)$ & \\
\hline De 45-30 & 1781 & $274(15,4)$ & & $246(13,8)$ & \\
\hline De 29-18 & 643 & $137(21,3)$ & & $111(17,3)$ & \\
\hline De $17-15$ & 11 & $3(27,3)$ & & $3(27,3)$ & \\
\hline \multicolumn{6}{|l|}{ Violencia en la niñez } \\
\hline NO & 1196 & $99(8,3)$ & $<0,001$ & $89(7,4)$ & $<0,001$ \\
\hline sí & 2713 & $418(15,4)$ & & $382(14,1)$ & \\
\hline \multicolumn{6}{|l|}{ Actitud favorable hacia la violencia } \\
\hline NO & 2911 & $336(11,5)$ & $<0,001$ & $313(10,8)$ & $<0,001$ \\
\hline sí & 958 & $178(18,6)$ & & $156(16,3)$ & \\
\hline \multicolumn{6}{|l|}{ Consumo excesivo de alcohol (último mes) } \\
\hline Nunca & 2157 & $223(10,3)$ & $<0,001$ & $213(9,9)$ & $<0,001$ \\
\hline Una vez & 1288 & $204(15,8)$ & & $171(13,3)$ & \\
\hline 3 o 4 veces & 384 & $74(19,3)$ & & $68(17,7)$ & \\
\hline 5 a más veces & 80 & $16(20)$ & & $19(23,8)$ & \\
\hline \multicolumn{6}{|l|}{ Grado de instrucción } \\
\hline Superior universitaria completa & 599 & $29(4,8)$ & $<0,001$ & $27(4,5)$ & $<0,001$ \\
\hline Superior no universitaria completa & 486 & $49(10,1)$ & & $51(10,5)$ & \\
\hline Universitaria o no universitaria incompleta & 329 & $45(13,7)$ & & $46(14)$ & \\
\hline Secundaria completa & 1366 & $204(14,9)$ & & $179(13,1)$ & \\
\hline Secundaria incompleta o menos & 1129 & $190(16,8)$ & & $168(14,9)$ & \\
\hline \multicolumn{6}{|l|}{ Ingreso mensual familiar } \\
\hline De S/.2000 (USD 750) o más & 321 & $10(3,1)$ & $<0,001$ & $12(3,7)$ & $<0,001$ \\
\hline Entre S/.1200 (USD 450) y S/.2000 (USD 750) & 552 & $51(8,5)$ & & $41(6,8)$ & \\
\hline Entre S/. 840 (USD 315) y S/.1200 (USD 450) & 1074 & $155(21,6)$ & & $154(12,5)$ & \\
\hline Menor de S/. 840 (USD 315) & 1455 & $301(17,1)$ & & $264(15,0)$ & \\
\hline \multicolumn{6}{|l|}{ Ciudades } \\
\hline Lima & 2288 & $280(12,2)$ & 0,003 & $248(10,8)$ & $<0,001$ \\
\hline Arequipa & 295 & $29(9,8)$ & & $25(8,5)$ & \\
\hline Huamanga & 221 & $31(14)$ & & $24(10,9)$ & \\
\hline Cusco & 249 & $48(19,3)$ & & $47(18,9)$ & \\
\hline Trujillo & 300 & $51(17)$ & & $52(17,3)$ & \\
\hline Callao & 257 & $43(16,7)$ & & $47(18,3)$ & \\
\hline Maynas & 299 & $35(11,7)$ & & $28(9,4)$ & \\
\hline
\end{tabular}

* Obtenido al comparar el porcentaje de personas que respondieron "si" fueron agresores/víctimas frente a las que respondieron "no".

durante el último mes $(n=80)$. Esto podría ocasionar que los intervalos de confianza de los OR de asociación de estas categorías con las variables resultado sean amplios, denotando imprecisión estadística.

La Tabla 2 presenta los resultados de asociación entre ser víctima de violencia de pareja y cada una de las variables independientes. El modelo no ajustado muestra que tanto el hombre como la mujer tienen la misma probabilidad de ser las víctimas de violencia $(\mathrm{OR}=0,9$; intervalo de confianza al 95\%, IC 95\%: 0,8-1,1; $p=0,558)$. Respecto a la edad, las personas menores de 45 años tienen mayor probabilidad de ser víctimas de violencia de pareja, presentándose incluso una tendencia que sugiere que a menor edad mayor probabilidad de ser víctima con OR que van de 2,9 a 6,7 y valores $p<0,007$. Asimismo, aquellas personas que sufrieron violencia física durante la niñez, tuvieron mayor probabilidad de ser víctimas de violencia de pareja (OR=2,0; IC 95\%: 1,6 - 2,6; $p<0,001$ ), lo mismo que aquellas personas que tuvieron una actitud favorable (en el cuartil superior) a la violencia (OR=1,6; IC 95\%: 1,3 - 2,0; $p<0,001)$. 
Respecto al consumo excesivo de alcohol en el último mes, estuvo fuertemente asociado con ser víctima de violencia de pareja, comparado con no haber consumido, habiendo una tendencia dosis-respuesta que sugiere que a mayor involucramiento en consumo de alcohol, mayor probabilidad de ser víctima (OR para estas categorías van de 1,4 a 2,8 conforme se incrementa el consumo, con valores $p<0,001$ para aquellos que consumieron en exceso tres o más veces en el último mes).
Respecto al nivel de educación, aquellos que tenían educación superior universitaria completa, tuvieron menor probabilidad de ser víctima de violencia de pareja comparados con las demás categorías educativas especificadas también en la Tabla 2 ( $p<0,001$ para todas las categorías comparadas). Además, aquellos que tenían un ingreso mensual familiar menor a S/. 2000 (aproximadamente, 750 USD), tenían mayor probabilidad de ser víctimas de este tipo

Tabla 2. Factores asociados al rol de víctima en violencia contra la pareja en condición de convivencia actual

\begin{tabular}{|c|c|c|c|c|c|c|}
\hline \multirow{2}{*}{ Variable } & \multicolumn{3}{|c|}{$\begin{array}{c}\text { Ser víctima } \\
\text { (modelo crudo) }\end{array}$} & \multicolumn{3}{|c|}{$\begin{array}{c}\text { Ser víctima } \\
\text { (modelo multivariado) }\end{array}$} \\
\hline & OR & IC $95 \%$ & p & OR & IC $95 \%$ & p \\
\hline \multicolumn{7}{|l|}{ Sexo } \\
\hline Femenino & 1 & -- & -- & 1 & -- & -- \\
\hline Masculino & 0,9 & $0,8-1,1$ & 0,558 & 0,9 & $0,7-1,2$ & 0,515 \\
\hline \multicolumn{7}{|l|}{ Edad (años) } \\
\hline De 60 a más & 1 & -- & -- & 1 & -- & -- \\
\hline De 59-46 & 1,6 & $1,0-2,6$ & 0,045 & 1,6 & $1,0-2,6$ & 0,060 \\
\hline De 45-30 & 2,9 & $1,9-4,4$ & $<0,001$ & 2,8 & $1,8-4,4$ & $<0,001$ \\
\hline De 29-18 & 3,7 & $2,4-5,9$ & $<0,001$ & 3,7 & $2,3-5,9$ & $<0,001$ \\
\hline De 17-15 & 6,7 & $1,7-27,0$ & 0,007 & 8,4 & $2,0-35,2$ & 0,004 \\
\hline \multicolumn{7}{|l|}{ Violencia en la niñez } \\
\hline NO & 1 & -- & -- & 1 & -- & -- \\
\hline sí & 2,0 & $1,6,-2.6$ & $<0,001$ & 1,9 & $1,5-2,4$ & $<0,001$ \\
\hline \multicolumn{7}{|l|}{ Actitud favorable hacia la violencia } \\
\hline NO & 1 & -- & -- & 1 & -- & -- \\
\hline sí & 1,6 & $1,3-2,0$ & $<0,001$ & 1,5 & $1,2-1,9$ & $<0,001$ \\
\hline \multicolumn{7}{|l|}{ Consumo excesivo de alcohol (último mes) } \\
\hline Nunca & 1 & -- & -- & 1 & -- & -- \\
\hline Una vez & 1,4 & $1,1-1,7$ & 0,002 & 1,3 & $1,1-1,7$ & 0,010 \\
\hline 3 o 4 veces & 2,0 & $1,5-2,6$ & $<0,001$ & 2,1 & $1,5-2,9$ & $<0,001$ \\
\hline 5 a más veces & 2,8 & $1,7-4,8$ & $<0,001$ & 2,9 & $1,6-5,1$ & $<0,001$ \\
\hline \multicolumn{7}{|l|}{ Grado de instrucción } \\
\hline Superior universitaria completa & 1 & -- & -- & 1 & -- & -- \\
\hline Superior no universitaria completa & 2,5 & $1,5-4,0$ & $<0,001$ & 2,0 & $1,2-3,3$ & 0,006 \\
\hline Universitaria o no universitaria incompleta & 3,4 & $2,1-5,7$ & $<0,001$ & 3,0 & $1,8-4,9$ & $<0,001$ \\
\hline Secundaria completa & 3,2 & $2,1-4,8$ & $<0,001$ & 2,6 & $1,7-4,0$ & $<0,001$ \\
\hline Secundaria incompleta o menos & 3,7 & $2,4-5,6$ & $<0,001$ & 3,5 & $2,2-5,3$ & $<0,001$ \\
\hline \multicolumn{7}{|l|}{ Ingreso mensual familiar } \\
\hline De S/.2000 (USD 750) o más & 1 & -- & -- & 1 & -- & -- \\
\hline Entre S/.1200 (USD 450) y S/.2000 (USD 750) & 1,9 & $1,0-3,6$ & 0,06 & 1,7 & $0,9-3,2$ & 0,135 \\
\hline Entre S/. 840 (USD 315) y S/.1200 (USD 450) & 3,7 & $2,0-6,7$ & $<0,001$ & 3,0 & $1,6-5,5$ & $<0,001$ \\
\hline Menor de S/. 840 (USD 315) & 4,6 & $2,5-8,2$ & $<0,001$ & 3,5 & $1,9-6,4$ & $<0,001$ \\
\hline \multicolumn{7}{|l|}{ Ciudades } \\
\hline Lima & 1 & -- & -- & 1 & -- & -- \\
\hline Arequipa & 0,8 & $0,5-1,2$ & 0,215 & 0,8 & $0,5-1,3$ & 0,454 \\
\hline Huamanga & 1,0 & $0,6-1,6$ & 0,993 & 0,9 & $0,6-1,5$ & 0,761 \\
\hline Cusco & 1,9 & $1,4-2,7$ & $<0,001$ & 2,3 & $1,6-3,4$ & $<0,001$ \\
\hline Trujillo & 1,7 & $1,2-2,4$ & 0,001 & 1,5 & $1,1-2,1$ & 0,019 \\
\hline Callao & 1,8 & $1,3-2,6$ & $<0,001$ & 1,7 & $1,2-2,5$ & 0,002 \\
\hline Maynas & 0,8 & $0,6-1,3$ & 0,438 & 0,8 & $0,5-1,2$ & 0,328 \\
\hline
\end{tabular}

*Modelo multivariado contiene sexo, edad, violencia en la niñez, actitud favorable a la violencia, intoxicación alcohólica, grado de instrucción y región; excepto cuando se estima el coeficiente de asociación (OR) entre ingreso familiar y la variable dependiente, modelo en que se incluyó todas las covariables, excepto grado de instrucción. Esto fue debido a la colinearidad que hay entre ingreso mensual familiar y grado de instrucción, $p<0,001$. 
de victimización $(p<0,01)$. Finalmente, se encontró que, comparado con Lima Metropolitana, aquellos que residían en Cusco y Callao tuvieron mayor probabilidad de ser víctimas de violencia ( $p<0,001$ para ambas ciudades), sin diferencias entre Lima y las otras ciudades participantes, aunque se ve cierta tendencia a mayores niveles para Trujillo, pero no llegó a ser significativa. Todas estas asociaciones se mantuvieron en el modelo saturado, que se ajustó por todas las variables consideradas.

En los modelos de interacción, al explorar la posibilidad de un efecto modificador que el sexo pueda tener en la asociación entre cada una de las variables independientes y ser víctima de violencia física, que justifique presentar los OR para hombres y mujeres por separado, todos los coeficientes de los términos multiplicativos resultaron no significativamente diferentes de cero (valores $p>0,1$ para todos los casos), por lo que no fue necesario el informe estratificado por sexo de los estimados de asociación.

En la Tabla 3 se presentan los resultados hallados respecto a variables asociadas con ser agresor en eventos de violencia de pareja. De manera similar a

Tabla 3. Factores asociados al rol de agresor en violencia contra la pareja en condición de convivencia actual.

\begin{tabular}{|c|c|c|c|c|c|c|}
\hline & \multicolumn{3}{|c|}{$\begin{array}{c}\text { Agresor } \\
\text { (modelo crudo) }\end{array}$} & \multicolumn{3}{|c|}{$\begin{array}{c}\text { Agresor } \\
\text { (modelo multivariado)* }^{*}\end{array}$} \\
\hline & OR & IC $95 \%$ & $\mathbf{p}$ & OR & IC $95 \%$ & $p$ \\
\hline \multicolumn{7}{|l|}{ Sexo } \\
\hline Femenino & 1 & -- & -- & 1 & -- & - \\
\hline Masculino & 0,8 & $0,7-1,0$ & 0,039 & 0,8 & $0,6-1,0$ & 0,046 \\
\hline \multicolumn{7}{|l|}{ Edad (años) } \\
\hline De 60 a más & 1 & -- & -- & 1 & -- & - \\
\hline De 59-46 & 1,9 & $1,2-3,1$ & 0,012 & 1,8 & $1,1-3,1$ & 0,019 \\
\hline De 45-30 & 4,0 & $2,5-6,3$ & $<0,001$ & 3,7 & $2,3-6,0$ & $<0,001$ \\
\hline De 29-18 & 5,9 & $3,6-9,6$ & $<0,001$ & 5,7 & $3,4-9,4$ & $<0,001$ \\
\hline De $17-15$ & 8,2 & $2,0-33,1$ & 0,003 & 9,2 & $2,2-39,9$ & $<0,001$ \\
\hline \multicolumn{7}{|l|}{ Violencia en la niñez } \\
\hline NO & 1 & -- & -- & 1 & -- & - \\
\hline sí & 2,0 & $1,6-2,5$ & $<0,001$ & 1,9 & $1,5-2,4$ & $<0,001$ \\
\hline \multicolumn{7}{|l|}{ Actitud favorable hacia la violencia } \\
\hline NO & 1 & -- & -- & 1 & -- & - \\
\hline sí & 1,7 & $1,4-2,1$ & $<0,001$ & 1,7 & $1,4-2,1$ & $<0,001$ \\
\hline \multicolumn{7}{|l|}{ Consumo excesivo de alcohol (último mes) } \\
\hline Nunca & 1 & -- & -- & 1 & -- & - \\
\hline Una vez & 1,6 & $<0,001$ & $1,3-2,0$ & 1,7 & $1,3-2,1$ & $<0,001$ \\
\hline 3 o 4 veces & 2,1 & $<0,001$ & $1,6-2,8$ & 2,4 & $1,7-3,3$ & $<0,001$ \\
\hline 5 a más veces & 2,2 & 0,007 & $1,2-3,8$ & 2,2 & $1,2-4,1$ & 0,008 \\
\hline \multicolumn{7}{|l|}{ Grado de instrucción } \\
\hline Superior universitaria completa & 1 & -- & -- & 1 & -- & - \\
\hline Superior no universitaria completa & 2,2 & $1,4-3,5$ & 0,001 & 1,7 & $1,1-2,9$ & 0,025 \\
\hline Universitaria o no universitaria incompleta & 3,1 & $1,9-5.1$ & $<0,001$ & 2,6 & $1,6-4,3$ & $<0,001$ \\
\hline Secundaria completa & 3,5 & $2,3-5,2$ & $<0,001$ & 2,8 & $1,8-4,2$ & $<0,001$ \\
\hline Secundaria incompleta o menos & 4,0 & $2,7-6,0$ & $<0,001$ & 3,7 & $2,4-5,6$ & $<0,001$ \\
\hline \multicolumn{7}{|l|}{ Ingreso mensual familiar } \\
\hline De S/.2000 (USD 750) o más & 1 & -- & -- & 1 & -- & - \\
\hline Entre S/.1200 (USD 450 y S/.2000 (USD 750) & 2,9 & $1,4-8,6$ & 0,003 & 2,6 & $1,3-5,2$ & 0,009 \\
\hline Entre S/. 840 (USD 315) y S/.1200 (USD 450) & 4,5 & $2,3-8,6$ & $<0,001$ & 3,6 & $1,9-7,1$ & $<0,001$ \\
\hline Menor de S/. 840 (USD 315) & 6,4 & $3,4-12,2$ & $<0,001$ & 4,9 & $2,6-9,4$ & $<0,001$ \\
\hline \multicolumn{7}{|l|}{ Ciudades } \\
\hline Lima & 1 & -- & -- & 1 & -- & - \\
\hline Arequipa & 0,8 & $0,5-1,2$ & 0,232 & 0,9 & $0,6-1,3$ & 0,524 \\
\hline Huamanga & 1,2 & $0,8-1,7$ & 0,441 & 1,1 & $0,7-1,7$ & 0,574 \\
\hline Cusco & 1,7 & $1,2-2,4$ & 0,002 & 2,1 & $1,5-3,0$ & $<0,001$ \\
\hline Trujillo & 1,5 & $1,1-2,0$ & 0,021 & 1,3 & $0,9-1,8$ & 0,193 \\
\hline Callao & 1,4 & $1,0-2,0$ & 0,041 & 1,3 & $0,9-1,9$ & 0,138 \\
\hline Maynas & 1,0 & $0,7-1,4$ & 0,791 & 0,9 & $0,6-1,3$ & 0,642 \\
\hline
\end{tabular}

*Modelo multivariado contiene sexo, edad, violencia en la niñez, actitud favorable a la violencia, intoxicación alcohólica, grado de instrucción y región, excepto cuando se estima el coeficiente de asociación (OR) entre ingreso familiar y la variable dependiente, modelo en que se incluyó todas las covariables, excepto grado de instrucción. Esto fue debido a la colinearidad que hay entre ingreso mensual familiar y grado de instrucción, p<0,001. 
lo visto para la víctima, en el modelo crudo, el sexo no estuvo asociado con mayor probabilidad de ser agresor $(p>0,01)$. Igualmente, los menores de 45 años tuvieron mayor probabilidad de ser agresores de una manera que tiende a ser mayor conforme más joven es la persona $(p<0,01)$. Aquellos que habían sufrido violencia física en la niñez y aquellos que tenían una actitud más positiva hacia la violencia, tuvieron más probabilidad de ser agresores (OR: 2,0 y 1,7, respectivamente, con valor $p<0,001$ para ambas asociaciones). El consumo excesivo de alcohol, por lo menos una vez en el último mes, también estuvo asociado con ser agresor, comparado con no haber consumido alcohol. Además, de la misma manera que con ser víctima, aquellos que concluyeron el nivel superior universitario o tenían un ingreso mensual familiar de más de S/. 2000 ( 750 USD), tuvieron menos probabilidad de agredir a su pareja, comparados con todos los demás grados académicos alcanzados o tenían un ingreso familiar de más de esa cantidad, respectivamente.

Además, y esto constituye una diferencia con lo que se encontró al analizar el rol de víctima, solo aquellos que residen en Cusco, tuvieron mayor probabilidad de ser agresores comparados con aquellos que residían en Lima Metropolitana. Todas estas asociaciones encontradas, se mantuvieron en el modelo saturado de ajuste, indicando que cada variable es predictora independiente de ser agresor, igual que en el caso de ser víctima. Los modelos de interacción por sexo para el rol de agresor tampoco dieron resultados significativos, por lo que el informe estratificado por sexo de los OR tampoco fueron necesarios $(p>0,1)$.

Finalmente, el tiempo de convivencia no resultó asociado ni con ser víctima, ni con ser el o la agresora, en los casos de violencia contra la pareja, tanto en el modelo crudo como en el ajustado. Asimismo, fue interesante notar que aproximadamente $70 \%$ de aquellos que fueron víctimas también fueron agresores/as (OR: 42,7; $p<0,001$ ).

\section{DISCUSIÓN}

El hallazgo más importante del presente estudio es que el patrón de características que predice quién es la víctima es muy similar al que predice quién es el agresor, en los casos de violencia contra la pareja. En general, ser menor de 45 años, el haber sufrido niveles altos de violencia durante la niñez, el tener una actitud favorable hacia la violencia, el consumir alcohol, el no tener un grado universitario, un ingreso mensual menor a 750 USD y el vivir en el Cusco, están asociados de manera fuerte e independiente tanto con ser la víctima como con ser el agresor en los casos de violencia contra la pareja. Es interesante, además, notar que el sexo y el tiempo de convivencia no aparecieron asociados con ninguno de ambos roles en este tipo de violencia. Todos estos resultados coinciden con lo encontrado en estudios que, en los últimos años, se han realizado en el ámbito internacional ${ }^{(9-13)}$.

Antes de pasar a una discusión de las implicancias de los resultados, mencionaremos las debilidades y fortalezas de este estudio. La primera limitación tiene que ver con su diseño transversal, que no permite establecer temporalidad entre factores, como el consumo de alcohol o una actitud favorable hacia la violencia, y el hecho de ser víctima o agresor en los casos de violencia contra la pareja. Sin embargo, respecto al consumo de alcohol, hay suficiente evidencia que refrenda su asociación causal con violencia de pareja. La asociación con actitud favorable hacia la violencia requiere futuros estudios que evalúen si las cogniciones a favor de la violencia preceden el primer acto violento o si, más bien, la persona que es violenta desarrolla cogniciones que justifican su violencia. El haber sufrido violencia física durante la niñez y, en la mayoría de casos, el nivel de educación alcanzado, son características que preceden los eventos de violencia de pareja del último año, lo que apoyaría una posible asociación causal.

Otra limitación es que, para este análisis secundario de los datos del estudio del CONASEC, no se pudo recuperar las ponderaciones de probabilidad de selección de los participantes, ni las variables de estratificación multinivel empleadas en la selección de la muestra. Esto no ha permitido que se haga un análisis estadístico para muestras complejas, lo que explica que no presentemos prevalencias poblacionales de cada una de las variables, sino porcentajes no-ponderados que dan cuenta de la frecuencia de cada variable en la muestra estudiada, la cual puede no coincidir con lo que ocurre en la población. Sin embargo, los análisis de asociación entre variables, que son el foco principal del presente estudio, son afectados en menor medida por este hecho. Específicamente, si bien es cierto que un diseño multinivel de muestreo crea muestras más homogéneas $y$, por ello, con mayor probabilidad de encontrar asociaciones significativas, la validez de la asociación se mantiene, especialmente si esta asociación tiene valores $p<0,01$. En el caso del presente estudio, el nivel de asociación de casi todas las variables tuvieron valores $p<0,001$, lo que da confianza de su validez.

Entre las fortalezas de este estudio tenemos que es poblacional, con selección probabilística, lo cual disminuye la posibilidad de sesgo de selección diferenciada respecto a cualquiera de las variables 
que se asocian al rol de víctima o agresor en los casos de violencia de pareja. De hecho, este es el tipo de sesgo que hace controversiales a los estudios realizados con individuos que llegan a ser atendidos en instituciones de salud, policiales o judiciales, ya que estas son generalmente mujeres y además pueden ser cualitativamente diferentes de aquellas que no llegan a buscar ayuda. Otra fortaleza es que se incluye tanto hombres como mujeres, como ha sido recomendado por el Ministerio de la Mujer en el Perú, sobrepasando la asunción restringida de que la violencia de pareja es solo un problema de la mujer. Estudiar todos los roles y ambos actores ayudará a un mejor conocimiento de este problema y así poder identificar mejor las acciones más costo-efectivas ${ }^{(14)}$

Tomando en cuenta las limitaciones y fortalezas, el presente estudio permite conocer características fácilmente identificables en la población, que permiten identificar grupos en mayor riesgo de violencia. Algunas de las características, como la edad, el sexo, la región de residencia o el ingreso familiar, son meramente predictivas, puesto que no es posible modificarlas desde la salud pública. Estas variables predictivas, que también aparecen asociadas con violencia contra la pareja en otros estudios ${ }^{(15-17)}$, serían muy útiles para identificar a los grupos de mayor riesgo, que son los que más se beneficiarían de medidas para disminuir la violencia doméstica.

Otras variables asociadas, como el consumo de alcohol, la actitud favorable o tolerancia a la violencia y el haber sido víctima de violencia física en la niñez, son hallazgos consistentes con la literatura y señalan factores potencialmente modificables desde la salud pública, preferentemente en combinación ${ }^{(18-22)}$.

Un hallazgo interesante es la probabilidad similar que tienen tanto hombres como mujeres en el desempeño de ambos roles, agresor y víctima, con una ligera mayor probabilidad en la mujer de ser agresora respecto al varón, lo cual ha sido documentado en otros estudios en el ámbito internacional ${ }^{(9,10)}$, aunque los varones tienen mayor probabilidad de causar lesiones ${ }^{(23)}$.Todo esto sugiere que las acciones para disminuir la violencia contra la pareja deben ser dirigidas tanto al hombre como a la mujer y asumir que ambos cumplen roles tanto de agresores como de víctimas, como lo sugiere nuestro hallazgo donde ambos roles tienen similares factores predictores y se presentan estrechamente correlacionados. Hacerlo de otra manera hace que las intervenciones tengan poca probabilidad de ser efectivas ${ }^{(24-26)}$. Por otra parte, estos resultados plantean el reto de estudiar con mayor profundidad la dinámica y participación de la mujer y el hombre en la violencia de pareja.
Finalmente, es de notar que varios de nuestros hallazgos desafían algunos supuestos en el área, que muchas veces dan sustento a investigaciones, políticas y programas de intervención. Por ejemplo, esperábamos que la probabilidad de ser víctima sería mayor en mujeres que en hombres, que la probabilidad de ser agresor sería mayor en hombres y que ambos indicadores serían muy altos en todas las localidades de la sierra, especialmente en Huamanga, dada la violencia política sufrida en dicha localidad durante la década de 1980 y parte de los 90 . Nada de esto pudo verificarse empíricamente en este estudio. Esto nos pone frente al desafío de continuar investigando este fenómeno en el contexto peruano, ya que aparecen más preguntas que son importantes de resolver para poder establecer medidas de control pertinentes.

En conclusión, este estudio muestra que las probabilidades de ser víctima o agresor son similares para ambos sexos. También encontramos que la exposición a la violencia a la niñez, tener una actitud favorable a la violencia, ser menor de 45 años, menor nivel de educación y de ingreso mensual familiar, así como también el consumo de alcohol son factores asociados con la violencia física contra la pareja. Geográficamente, el Cusco es la ciudad que tiene una asociación significativa con ejercer o ser víctima de violencia conyugal. Entender las complejidades de la violencia de pareja puede permitir el desarrollo de políticas públicas integrales que aborden el problema de una manera más efectiva y eficiente.

\section{AGRADECIMIENTOS}

A la Secretaría Técnica de la CONASEC por proporcionar la base de datos para este estudio.

\section{Contribuciones de autoría}

FF, RR, EG Y AG participaron de la ideación de estudio. FF y $\mathrm{RR}$ llevaron a cabo los análisis. FF escribió el primer borrador del artículo. RR, EG Y AG contribuyeron significativamente con la interpretación de los resultados y la escritura del manuscrito. Todos los autores toman responsabilidad de la publicación.

\section{Fuentes de financiamiento}

Este trabajo se llevó a cabo con apoyo del Programa de Trauma y Salud Global con el apoyo de la iniciativa de Investigación en Salud Mundial, una asociación dedicada al financiamiento de la investigación entre los Institutos Canadienses de Investigación en Salud, la Agencia Canadiense para el Desarrollo Internacional, el Ministerio de Salud de Canadá, el Centro Internacional de Investigaciones para el Desarrollo, y la Agencia de Salud Pública de Canadá.

\section{Conflictos de interés}

Los autores declaran no tener conflictos de interés. 


\section{REFERENCIAS BIBLIOGRÁFICAS}

1. United Natios. Declaration on the elimination of violence against women. A/RES/48/104. [Internet]. New York: UN; c2012 [citado el 9 de enero del 2012]. Disponible en: http:// www.un.org/documents/ga/res/48/a48r104.htm

2. Flury $M$, Nyberg $E$, Riecher-Rössler A. Domestic violence against women: Definitions, epidemiology, risk factors and consequences. Swiss Med Wkly. 2010;140:w13099.

3. Garcia-Moreno C, Jansen HA, Ellsberg M, Heise L, Watts $\mathrm{CH}$; WHO Multi-country Study on Women's Health and Domestic Violence against Women Study Team. Prevalence of intimate partner violence: findings from the $\mathrm{WHO}$ multicountry study on women's health and domestic violence. Lancet. 2006;368(9543):1260-9.

4. Instituto Nacional de Estadística e Informática (INEI). Encuesta Demográfica y de Salud familiar 2010 [Internet] Lima: INEI; 2011 [citado el 9 de enero del 2012]. Disponible en: $\quad$ http://proyectos.inei.gob.pe/endes/endes2010/ resultados/caratula.htm

5. Güezmes A, Palomino N. Violencia sexual y física contra las mujeres en el Perú. Lima: Flora Tristan; 2002.

6. Krug EG, Mercy JA, Dahlberg LL, Zwi AB. The world report on violence and health. Lancet. 2002;360(9339):1083-8.

7. Gushiken A, Costa G, Romero C, Privat C. Perú: Nueva Publicación de Ciudad Nuestra ¿Quiénes son asesinad@s en Lima? ¿Cómo, cuándo y por qué? [Internet]. Washington, DC: Woodrow Wilson International Center for Scholars; c2012 [citado el 12 de enero del 2012]. Disponible en: http:// scela.wordpress.com/2010/04/18/peru-nueva-publicacionde-ciudad-nuestra-\%c2\%bfquienes-son-asesinads-enlima-\%c2\%bfcomo-cuando-y-por-que-alfonso-gushikengino-costa-carlos-romero-y-catherine-privat/

8. Fournier $M$, de los Ríos $R$, Orpinas $P$, Piquet-Carneiro $L$. Multicenter Study on Cultural Attitudes and Norms towards Violence (ACTIVA project): methodology. Rev Panam Salud Publica. 1999;5(4-5):222-31.

9. Bair-Merritt MH, Crowne SS, Thompson DA, Sibinga E, Trent M, Campbell J. Why do women use intimate partner violence? A systematic review of women's motivations. Trauma Violence Abuse. 2010;11(4):178-89.

10. Carrado M, George MJ, Loxam E, Jones L, Templar D. Aggression in British heterosexual relationships: a descriptive analysis. Aggressive Behavior. 1996;22(6):40115.

11. Orpinas P. Who is violent?: factors associated with aggressive behaviors in Latin America and Spain. Rev Panam Salud Publica. 1999;5(4-5):232-44.

12. Moreno Martín F. Violence between couples. Rev Panam Salud Publica. 1999;5(4-5):245-58.

13. Muñoz E, Gutiérrez MI, Arango OA, Guerrero R. Creencias, Actitudes y Prácticas sobre Violencia en Bogotá, Cali y Medellín. Línea de Base. [Internet]. Santiago de Cali: Ministerio de Protección Social, Universidad del Valle, Instituto CISALVA; 2004 [citado el 12 de enero del 2012]. Disponible en: http://grupocisalva.univalle.edu.co/ publicaciones/documentos/linea_de base.pdf
14. Melton HC, Sillito CL. The role of gender in officially reported intimate partner abuse. J Interpers Violence. 2011. [Epub ahead of print]

15. da Fonseca RM, Leal AE, Skubs T, Guedes RN, Egry EY. Domestic violence against women from the perspective of the community health agent. Rev Lat Am Enfermagem. 2009;17(6):974-80.

16. Selic P, Pesjak K, Kersnik J. The prevalence of exposure to domestic violence and the factors associated with co-occurrence of psychological and physical violence exposure: a sample from primary care patients. BMC Public Health. 2011;11:621.

17. Vives-Cases C, Gil-González D, Carrasco-Portiño M, Alvarez-Dardet C. Systematic review of studies on the socioeconomic status of men who batter their intimate partners. Gac Sanit. 2007;21(5):425-30.

18. Zaleski M, Pinsky I, Laranjeira R, Ramisetty-Mikler $S$, Caetano R. Intimate partner violence and alcohol consumption. Rev Saude Publica. 2010;44(1):53-9.

19. Zhan W, Shaboltas AV, Skochilov RV, Kozlov AP, Krasnoselskikh TV, Abdala N. Alcohol misuse, drinking contexts and intimate partner violence in St. Petersburg, Russia: results from a cross-sectional study. BMC Public Health. 2011;11:629.

20. de Oliveira JB, Lima MCP, Simão MO, Cavariani MB, Tucci AM, Kerr-Corrêa F. Violence between intimate partners and alcohol use: prevalence and associated factors. Rev Panam Salud Publica. 2009;26(6):494-501.

21. Gil-González D, Vives-Cases C, Ruiz MT, Carrasco-Portiño $\mathrm{M}$, Alvarez-Dardet $\mathrm{C}$. Childhood experiences of violence in perpetrators as a risk factor of intimate partner violence: a systematic review. J Public Health (Oxf). 2008;30(1):14-22.

22. Gil-González D, Vives-Cases C, Alvarez-Dardet C, Latour-Pérez J. Alcohol and intimate partner violence: do we have enough information to act? Eur J Public Health. 2006;16(3):279-85.

23. Archer J. Sex differences in aggression between heterosexual partners: a meta-analytic review. Psychol Bull. 2000;126(5):651-80.

24. Wathen $\mathrm{CN}$, MacMillan $\mathrm{HL}$. Interventions for violence against women: scientific review. JAMA. 2003;289(5):589600.

25. Ramsay J, Carter Y, Davidson L, Dunne D, Eldridge S, Feder $\mathrm{G}$, et al. Advocacy interventions to reduce or eliminate violence and promote the physical and psychosocial wellbeing of women who experience intimate partner abuse. Cochrane Database Syst Rev. 2009;(3):CD005043.

26. Smedslund G, Dalsbø TK, Steiro AK, Winsvold A, ClenchAas J. Cognitive behavioural therapy for men who physically abuse their female partner. Cochrane Database Syst Rev. 2007;(3):CD006048.

Correspondencia: Fabián Fiestas

Dirección: Cápac Yupanqui 1400, Lima 11, Perú

Teléfono: (51) 985765743

Correo electrónico: ffiestas@epi.msu.edu 